\title{
Majorana bound states in the finite-length chain
}

\author{
A.A. Zvyagin \\ Max-Planck-Institut für Physik komplexer Systeme, Noethnitzer Str. 38, D-01187, Dresden, Germany \\ B.I. Verkin Institute for Low Temperature Physics and Engineering of the National Academy of Sciences of Ukraine \\ 47 Lenin Ave., Kharkov 61103, Ukraine \\ E-mail: zvyagin@ilt.kharkov.ua
}

Received May 15, 2015, published online June 23, 2015

\begin{abstract}
Recent experiments investigating edge states in ferromagnetic atomic chains on superconducting substrate are analyzed. In particular, finite size effects are considered. It is shown how the energy of the Majorana bound state depends on the length of the chain, as well as on the parameters of the model. Oscillations of the energy of the bound edge state in the chain as a function of the length of the chain, and as a function of the applied voltage (or the chemical potential) are studied. In particular, it has been shown that oscillations can exist only for some values of the effective potential.
\end{abstract}

PACS: $71.10 . P m$ Fermions in reduced dimensions;

74.20. $-\mathbf{z}$ Theories and models of superconducting state.

Keywords: Majorana edge states, topological superconductivity.

Majorana fermions have attracted a great attention due to their main features, comparing to usual Dirac's fermions. Majorana fermions are particles, identical to their own antiparticles. The combination of a pair of Majorana fermions can form a Dirac fermion. Also, Majorana fermions satisfy a non-Abelian statistics, different from the Fermi-Dirac one. It permitted [ 1 to point out potential use of Majorana fermions as qubits (elementary cells of quantum computers) in fault-tolerant topological computations. Their another usefulness in quantum computation is caused by the fact that spatially separated pairs of Majorana fermions can encode information as a highly nonlocal qubit, minimizing that way the decoherence of the quantum computer. This is why, the search for Majorana fermions is among the most prominent tasks for physicists.

For conventional superconductors with $s$-wave pairing, superpositions of electrons and holes carrying opposite spin are different from Majorana's construction. Majorana fermions can emerge in special superconductors, in which electrons and holes with the same value of spin can be paired. Kitaev has proposed to use zero-energy Majorana bound states at the opposite edges of a superconducting chain for the construction of a composite nonlocal qubit [2]. He has considered the simplest model of one-dimensional (1D) spinless fermions with pairing. 1D systems are important for the potential use in the quantum computation because: (i) their quantum features are enhanced (comparing to sys- tems of higher dimensionality) due to the 1D peculiarity in the density of states, and (ii) theorists can obtain exact (nonperturbative), often analytic results in 1D. The latter can be used for comparison of experimental data with theoretical predictions, very important for probabilistic quantum computation.

For the realization of Kitaev's scenario several 1D superconducting systems were proposed: low-dimensional topological insulators [3], quantum wires with the strong spin-orbit coupling in the external magnetic field in the vicinity of the standard $s$-wave superconductor [4], and ferromagnetic chains on the surface of such a superconductor [5]. Experimental attempts to implement the semiconductor wire proposal were successful to observe the zerobias peak in the tunneling spectroscopy studies of hybrid superconductor-semiconductor nanostructures [6]. That detected peak was explained as the manifestation of the Majorana edge states at zero-energy in a quantum wire. However, observed zero-bias peaks can be also explained as the manifestation of the disorder, or the Kondo resonance [7]. Moreover, the disadvantage of tunneling studies in semiconductor wires is that they lack the ability to spatially resolve zero bias peak features together with the zero-energy of the observed quantum state - to demonstrate that they are really connected with the edge Majorana states. This is why, ferromagnetic chains in the vicinity of the $s$-wave superconductor (e.g., on its surface) promise 
more advantages in observation and manipulation with edge Majorana modes [5].

Recently zero-energy edge states were observed in ferromagnetic iron atomic chains (from 30 up to $500 \AA$ ) on the surface of superconducting lead using spectroscopic imaging technique [8]. Excitations in the bulk of chains were gapped due to the proximity effect of the $s$-wave superconducting lead substrate, and the strong spin-orbit coupling in $\mathrm{Pb}$ yielded the effective $p$-wave pairing. The spin-orbit coupling (necessary for the transformation of the $s$-wave pairing of the substrate to the effective $p$-pairing in the chain [9]) was estimated to be $\sim 100 \mathrm{meV}$ in Ref. 8, comparing to the much smaller value in semiconducting quantum wires $(\sim 0.05 \mathrm{meV})$ [6]. Zero bias peaks disappeared over a distance of $10 \AA$ from the edges of studied magnetic chains. The Kondo resonance as the explanation of the zero-energy states was excluded: The application of the external magnetic field (up to $0.1 \mathrm{~T}$ ) suppressed superconductivity, but did not suppress the zero bias peak, as was expected from the theory of the Kondo effect. Also, in accordance with Anderson's theorem, defects of the substrate did not produce in-gap states. Finally, spin-polarized scanning tunneling microscope measurements did not show substantial magnetization changes at the edges of chains, which excludes the suppression of the pairing at the edges of chains, hence producing zero-energy peaks.

Despite such a great progress in the observation of (Majorana) edge modes in ferromagnetic atomic chains on a superconducting surface, some loose ends remain to contend with [10]. In fact, it was clear from the estimates $[2,11]$ that Majorana edge fermions in finite-length (of the size $L$ ) quantum chains have to hybridize to form a conventional Dirac fermion with the energy proportional to $\exp (-L / \xi)$, where $\xi$ is the coherence length of the superconducting chain. Obviously, for large lengths $L$ and small enough coherence lengths, $L \gg \xi$, the energy of such a bound state goes to zero. However, in the experiment [8] the energy gap of the induced superconductivity was very small, about $0.2 \mathrm{meV}$, comparing to the bandwidths of order of $0.2-0.7 \mathrm{eV}$. That implies (together with the not so pronounced gap structure) long coherence lengths. In this situation, the opposite case $\xi \gg L$ can persist, which means that Majorana edge states strongly hybridize to form a Dirac fermion with essentially nonzeroenergy. The short-distance physics of open superconducting chains with edge Majorana modes is not yet fully understood [10].

Then, the goal of the present study follows: to investigate analytically how the energy of bound edge states in open superconducting chains depends on the length. In the present work we consider first the Kitaev chain model of the finite length. We show how the energy of the bound state depends on the length of the chain, as well as on the parameters of the model, like the bandwidth, the pairing amplitude, and the potential. Depending on the value of the potential, the wavenumbers of the bound state can be either totally imaginary, or complex. The real part of the wavenumber implies standing wave, which magnitude decays exponentially (caused by the imaginary part of the wavenumber) from the distance of the edge. Hence, oscillations of the energy of the bound edge state in the Kitaev chain as a function of the length of the chain have to exist. Importantly, such oscillations can exist only for some region of the values of the effective voltage. Oscillations of the energy of such a bound state as a function of the applied voltage (or change of the chemical potential) can be also observed. We have mapped the effective Hamiltonian of the ferromagnetic metallic chain in the vicinity of the $s$ wave superconducting substrate to the Kitaev model with modified pairing amplitude and effective potential, and checked whether predicted effects of the finite size of the chain can be observed in potential experiments on such ferromagnetic atomic chains.

To start with, let us consider the model, which describes experiments on metallic magnetic chains situated on the $s$-wave superconductor substrate. The Hamiltonian of such a system can be written in the form [8]

$$
\begin{gathered}
\mathcal{H}_{m}=\sum_{j, j^{\prime}, \gamma, \gamma^{\prime}, \sigma} t_{\gamma, \gamma^{\prime}} c_{j \gamma \sigma}^{\dagger} c_{j^{\prime} \gamma^{\prime} \sigma}+\sum_{j, \gamma, \sigma} U_{\mathrm{eff}} c_{j \gamma \sigma}^{\dagger} c_{j \gamma \sigma}+ \\
+i t_{R} \sum_{j, j^{\prime}, \sigma, \sigma^{\prime}} \hat{z} \cdot\left(\hat{d}_{j j^{\prime}} \times s_{\sigma \sigma^{\prime}}\right) c_{j, \sigma}^{\dagger} c_{j, \sigma^{\prime}}+\lambda_{s o} \mathbf{L} \cdot \mathbf{s}+ \\
+\Delta_{\text {in }} \sum_{j, j^{\prime}, \gamma, \gamma^{\prime}}\left(c_{j \gamma \uparrow}^{\dagger} c_{j^{\prime} \gamma^{\prime} \downarrow}^{\dagger}+\text { h.c. }\right)-J \mathbf{m} \cdot \mathbf{s}
\end{gathered}
$$

where $c_{j \gamma \sigma}^{\dagger}\left(c_{j \gamma \sigma}\right)$ creates (destroys) an electron belonging to the $\gamma$-orbital (for the magnetic metallic chain for $3 d$ orbitals we have three bands with the magnetic quantum numbers 0,1 and 2) with the spin projection $\sigma=\uparrow, \downarrow$ at the site $j\left(j, j^{\prime}\right.$ denote nearest and, possibly, nextnearest neighbors), $t_{\gamma, \gamma^{\prime}}$ are the hopping integrals in the Slater-Koster approximation for the tight-binding Hamiltonian, $J$ is the exchange integral, $\mathbf{m}$ is the magnetization per site of the ferromagnetic chain (it is directed perpendicular to the chain), $\mathbf{s}$ is the spin operator, $\mathbf{L}$ is the operator of the orbital moment, $\lambda_{s o}$ is the on-site spin-orbit coupling constant for $3 d$ orbitals of the magnetic ion of the chain, $t_{R}$ is the Rashba spin-orbit coupling due to the hybridization between $3 d$ orbitals of ions of the metallic magnetic chain and $p$-orbitals of the superconducting substrate, $\Delta_{\text {in }}$ describes the proximity-induced pairing potential caused by the interaction of the electrons of the metallic chain with the $s$-wave superconducting substrate, and, finally, $U_{\text {eff }}$ defines the effective on-site potential. Only minority spin bands for Fe chains [8] cross the Fermi level with the hopping integrals of order of $0.6702 ; 0.5760$ and $0.1445 \mathrm{eV}$. Then, for each band, for which the topological 
superconductivity is expected to exist, we can write the following approximate Hamiltonian

$$
\begin{gathered}
\mathcal{H}_{\mathrm{appr}}=\sum_{j}\left(\sum _ { \sigma } \left[\left([w-\mu+U] c_{j \sigma}^{\dagger} c_{j \sigma}-\right.\right.\right. \\
-\frac{1}{2} \sum_{\sigma^{\prime}}\left(w-i \alpha \sigma_{y}\right) c_{j, \sigma^{\dagger}}^{\dagger} c_{j+1, \sigma^{\prime}}+\text { h.c. }+ \\
\left.\left.\left.+J \sigma_{z} c_{j \sigma}^{\dagger} c_{j \sigma^{\prime}}\right)\right]+\Delta_{\text {in }}\left(c_{j \uparrow}^{\dagger} c_{j+1 \downarrow}^{\dagger}+\text { h.c. }\right)\right),
\end{gathered}
$$

where $w$ is the effective width of the band for the considered minority spin band (one of the mentioned above values of $\left.t_{\gamma, \gamma^{\prime}}\right), U$ is the sum of the applied external voltage and internal potential (e.g., the on-site scalar potential induced by the magnetic atoms in the chain), and $\alpha$ is the effective spin-orbit coupling constant (both on-site and Rashba terms can contribute). The Hamiltonian is similar (up to the re-definition of constants) to the Hamiltonian of the quantum wire with the strong spin-orbit coupling in the external magnetic field (here the effective molecular field $J / 2$ of the magnetic chain plays the role of the external field), and with the proximity-induced pairing $\Delta_{\text {in }}$, considered [4] in the long-wave limit. First, let us diagonalize the $\Delta_{\text {in }}$-independent part of the Hamiltonian $H_{\text {appr }}$ by the unitary transformation (turning spins about the $x$ axis). After such a turn the effective $p$-wave pairing between electrons with the same projection of the spin takes place, which is proportional to $\Delta_{\text {in }} \alpha / J$. Then, as it was pointed out [12], we can project out the upper unoccupied band, which is legitimate for $J \gg \Delta_{\text {in }}$ (it is the case for the experiment in [8], see below). The effective Hamiltonian for the remaining band (in which edge Majorana fermions can exist) has the form of Eq. (3) with $\Delta \rightarrow \Delta_{\text {eff }}=2 \Delta_{\text {in }} \alpha / J$ and $V \rightarrow V_{\text {eff }}=\mu+(J / 2)-U-w$. Hence, the most important part of the effective Hamiltonian, which describes the behavior of the electrons of the ferromagnetic metallic chain in the vicinity of the $s$-wave superconductor with the strong spin-orbit coupling, is equivalent to the Hamiltonian of the Kitaev chain with the renormalized effective potential and effective pairing amplitude.

Now let us turn to the Kitaev chain model [2]. The Hamiltonian of the model can be written as [9]

$$
\mathcal{H}_{K}=-\frac{1}{2} \sum_{j=1}^{L-1}\left[w d_{j}^{\dagger} d_{j+1}+\Delta d_{j}^{\dagger} d_{j+1}^{\dagger}+\text { h.c. }\right]-V \sum_{j=1}^{L} d_{j}^{\dagger} d_{j},
$$

where $w$ and $\Delta$ are the hopping and pairing amplitudes, $V$ is the effective potential (the combination of the chemical potential and the applied external and internal potentials), $L$ is the length of the chain (in units of inter-atomic distances), and the operators $d_{j}\left(d_{j}^{\dagger}\right)$ destroy (create) a (Dirac) fermion in the site $j$. Here we consider the case of real $\Delta$ (the situation with complex values of $\Delta$ can be studied in a similar way). Suppose the following relation holds: $w, \Delta>0$ and $V \geq 0$ with $\Delta \leq w$ (other cases can be studied in a similar way). The ground state of the open chain is two-fold degenerate in the limit $L \rightarrow \infty$, see Refs. 2, 13. In one of two degenerate sets of the ground state eigenstates edge Majorana states persist [2,13], which can form a nonlocal Dirac fermion with zero-energy. Here we are looking for such a bound state for the finite-length chain.

The details of the solution of the stationary Schrödinger equation with the Hamiltonian $\mathcal{H}_{K}$ for eigenstates for the finite open Kitaev chain are similar to the one for spin-1/2 chains [14]. The Hamiltonian $\mathcal{H}_{K}$ can be diagonalized, i.e., presented in the diagonal form

$$
\mathcal{H}_{K}=\sum_{k} \varepsilon_{k} \eta_{k}^{\dagger} \eta_{k}+\text { const }
$$

where $\eta_{k}^{\dagger}\left(\eta_{k}\right)$ creates (destroys) a fermion with the wavenumber $k$ (that parametrizes all eigenfunctions), and $\varepsilon_{k} \geq 0$ is the energy of the eigenstate with the wavenumber $k$. For the cyclic chain for any $L$ any excitation has a gap, which for $V \leq\left(w^{2}-\Delta^{2}\right) / w$ is equal to

$$
G a p=\frac{\Delta \sqrt{w^{2}-\Delta^{2}-V^{2}}}{\sqrt{w^{2}-\Delta^{2}}},
$$

and for $V \geq\left(w^{2}-\Delta^{2}\right) / w$ is equal to

$$
\text { Gap }=|w-V| \text {. }
$$

The original Kitaev's Hamiltonian gets its diagonal form with the help of the unitary (Bogolyubov) transformation

$$
\begin{aligned}
& \eta_{k}=\sum_{j} u_{k j} d_{j}+v_{k j} d_{j}^{\dagger}, \\
& \eta_{k}^{\dagger}=\sum_{j} u_{k j} d_{j}^{\dagger}+v_{k j} d_{j},
\end{aligned}
$$

where the coefficients of the Bogolyubov transformation are

$$
2 u_{k j}=\varphi_{k j}+\psi_{k j}, \quad 2 v_{k j}=\varphi_{k j}-\psi_{k j} .
$$

For the chain consisting of $L$ sites $\varphi_{k}$ and $\psi_{k}$ are $L$-component vectors. Those vectors satisfy the equations

$$
\begin{aligned}
\hat{C} \hat{C}^{\dagger} \varphi_{k}= & \varepsilon_{k}^{2} \varphi_{k}, \quad \hat{C}^{\dagger} \varphi_{k}=\varepsilon_{k} \psi_{k}, \\
& \hat{C} \psi_{k}=\varepsilon_{k} \varphi_{k} .
\end{aligned}
$$

Here the matrix elements of $\hat{C}$ are

$$
C_{n, m}=-V \delta_{n, m}-\frac{w-\Delta}{2} \delta_{n, m-1}-\frac{w+\Delta}{2} \delta_{n, m+1} .
$$

There exist exactly $L$ roots of Eqs. (9) for wavenumbers, which parametrize eigenvalues and eigenfunctions. We know that eigenstates for the open chain are standing waves. Then the general form for the components of the $L$-component eigenvector $\varphi_{k}$ (analogously for $\psi_{k}$ ) can be looked for (for $j=1, \ldots, N)$ as 


$$
\varphi_{k j}=A_{1} \mathrm{e}^{i k_{1} j}+A_{2} \mathrm{e}^{-i k_{1} j}+A_{3} \mathrm{e}^{i k_{2} j}+A_{4} \mathrm{e}^{-i k_{2} j}
$$

with complex wavenumbers $k_{1,2}$. The coefficients $A_{1,2,3,4}$ are normalization constants. Notice that for the odd values of $L$ for $V=0$ we have $\operatorname{det} \hat{C}=0$, so that there exists a zero eigenvalue of the matrix $\hat{C} \hat{C}^{\dagger}$. Obviously, the wave number $k_{1,2}$ also satisfy the equation

$$
\varepsilon_{k_{1}}=\varepsilon_{k_{2}}
$$

Real values of $k_{1,2}$ describe the band eigenstates and complex ones describe bound (edge) states. Two sets of eigenfunctions for the considered open chain satisfy Eqs. (9). For example, for even $L$ and $V=0$ the components $\varphi_{j k}$ are nonzero (they are proportional to $\sin (j k) \sqrt{\sin (2 k) / \sin [2(L+1) k]}$, where $j=0,2,4, \ldots, L)$ for even sites of the chain and they are zero for odd sites of the chain for the first set of eigenstates. For $\psi_{j k}$ nonzero components are related to the odd sites of the chain (proportional to $\sin [(L-j) k] \sqrt{\sin (2 k) / \sin [2(L+1) k]}$, where $j=0,2, \ldots, L)$ for the first set in this case. For the second set the situation is opposite: even components of $\psi_{j k}$ and odd components of $\varphi_{j k}$ are nonzero, while other components are zero. This is why, if bound eigenstate exists, then its wave function decays exponentially with distances from each edge of the chain for the first and the second set of eigenfunctions. The energy of the bound eigenstate is zero in the thermodynamic limit $L \rightarrow \infty$, hence two sets of eigenfunctions are degenerate. It is, in general, not true for the finite $L$.

Let us concentrate on bound (edge) states. The wave functions of these states decay exponentially with the distance from edges. After some standard calculations one can show that for large enough $L$ there are $L-1$ real solutions for wavenumbers, which parametrize all eigenstates, and one complex solution (bound state) for $V \leq w$. (For $V>w$ one has $L$ real solutions for wavenumbers of band eigenstates.) We study long enough Kitaev's chain $L>4$ (in what follows we will consider only the main corrections for large $L$ ). For relatively small values of the effective potential, $0<V \leq F$ the wavenumbers of the edge bound states have nonzero real part $k$. Here for $L \gg 1$ one has for the critical value of the potential $V$,

$$
F=\sqrt{w^{2}-\Delta^{2}}-\frac{\Delta^{4}}{8(L+1) w^{2}(w+\Delta)}\left(\frac{w-\Delta}{w+\Delta}\right)^{L} .
$$

In the main order in $L$ for the energy of the bound state one gets (cf. Ref. 11)

$$
\varepsilon_{b}=G|\sin [(L+1) k]|\left(\frac{w-\Delta}{w+\Delta}\right)^{(L+1) / 2},
$$

where

$$
G=\frac{2 \Delta\left(w^{2}-V^{2}\right)}{w\left|w^{2}-\Delta^{2}-V^{2}\right|^{1 / 2}},
$$

and the value of $k$ is determined from $\cos k=V / F$. Notice that the nonzero real part of the wavenumber of the bound state, $k \neq 0$, exists only for $V \neq 0$. For the limiting cases $V=0$, and for $V \neq 0$ and $w=\Delta$, the real part is zero, $k=0$.

On the other hand, for $F \leq V \leq w$ wavenumbers for the edge bound states are only imaginary. For this region of parameters we have (cf. Ref. 11)

$$
\varepsilon_{b}=G \sinh [(L+1) p]\left(\frac{V+\sqrt{V^{2}-w^{2}+\Delta^{2}}}{(w+\Delta) \exp (p)}\right)^{L+1},
$$

where the value of $p$ is determined from $\cosh p \equiv$ $\equiv \cosh \left[\left(p_{1}+p_{2}\right) / 2\right]=V / F$. One can check that for $V=F$ the first solution for the bound state transforms to the second one. One can see from Eqs. (14) and (16) that the energy of the (Majorana) bound state is equal to zero (i.e., the exact degeneracy of two ground eigenstates persists), $\varepsilon_{b}=0$, only for $L \rightarrow \infty$, while for long, however finite lengths of the chain, $L$, the eigenvalue for the bound state become nonzero according to the law $\varepsilon_{b} \sim \exp (-L / \xi)$, cf. Refs. 2, 11. The correlation length $\xi$ depends on the values of the parameters of the Hamiltonian (the hopping and pairing amplitudes, $w$ and $\Delta$ ) and on the effective potential $V$. It turns out that for $V \leq F$ the correlation length $\xi=\ln [(w+\Delta) /(w-\Delta)]$ does not depend on the value of the effective potential, while for large values of the latter, for $V \geq F$, the correlation length is equal to $\xi=2 p+\ln \left[(w+\Delta) / V+\sqrt{V^{2}-w^{2}+\Delta^{2}}\right]$, i.e., it becomes $V$-dependent. We emphasize that for small enough values of the applied potential $V \leq F$ for each value of the length of the chain $L$ there exists the discrete set of the values of $V$ at which the exact degeneracy, $\varepsilon_{b}=0$ takes place. Namely, for

$$
V_{n}=F \cos [\pi n /(L+1)], n=1,2, \ldots, L / 2,
$$

the energy of the bound state is zero, and the exact degeneracy of two sets of ground state eigenstates is restored for the finite-length Kitaev chain. It would be interesting to check experimentally the possibility of those values of the effective potential for large enough quantum chains, in which zero-energy Majorana bound states exist. Notice the main differences in our results comparing to the ones, obtained for quantum semiconductor chains [11]: Oscillations of the bound state energy exist only if the effective potential is smaller than $F$, and in that range of parameters the correlation length does not depend on $V_{\text {eff }}$, unlike Ref. 11.

Now, the exchange integral for Fe chains on $\mathrm{Pb}$ substrate was approximately $2.4 \mathrm{eV}$. The on-cite spin-orbit coupling constant was $\lambda_{\text {so }} \sim 60 \mathrm{meV}$ for chains and 
$0.6 \mathrm{eV}$ for zigzag chains, comparing to the Rashba coupling $t_{R}=0.05 \mathrm{eV}$. Finally, the pairing $\Delta_{\text {in }}$ was estimated as $2-3 \mathrm{meV}$, see Ref. 8. The topological superconductivity (i.e., the case with the Majorana topological number is equal to -1 , for which Majorana edge states can exist) is expected for many values of the chemical potential [8], in particular for $J=2.4 \mathrm{eV}$ we can use the values of $|\mu|$ roughly between 0.1 and $2.5 \mathrm{eV}$. This is why, we can use our results for the finite-length Kitaev chain for the estimation of the effect of the finite length for the energy of the Majorana bound state in the ferromagnetic chain with the induced $p$-wave superconductivity [8].

Figure 1 shows how the energy of the bound state depends on the length of the chain (here we used $V_{\text {eff }}=$ $=0.1 \mathrm{meV}$ ). Let us analyze how the energy of the bound state depends on the value of the effective potential for the fixed length of the chain. The value of the effective potential $V_{\text {eff }}$ can be changed not only by application of the external voltage (with relatively small energies), but also by changing the chemical potential. The results are presented in Fig. 2. Large values of $V_{\text {eff }}$ are possible if one takes into account that it is determined as $V_{\text {eff }}=\mu+(J / 2)-U-w$. We see the clear difference between the values of the energy of the bound states in the finite chain for small and large values of the effective potential. Calculated values of the bound state energy can be observed in experiments using, e.g., the scanning tunneling spectroscopy.

Summarizing, we have considered the situation of recent experiments on magnetic atomic chains on $s$-wave superconducting substrate that observed Majorana edge

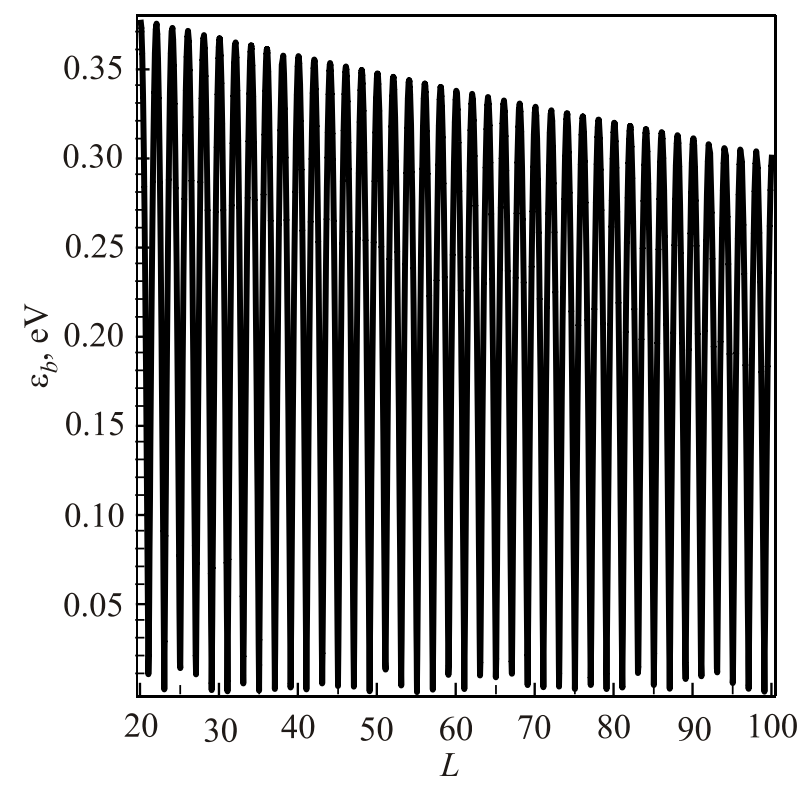

Fig. 1. The dependence of the energy of the bound state $\varepsilon_{b}$ in the Kitaev chain on the length of the chain $L$ (in units of inter-atomic distances in the chain). The following parameters are used: the bandwidth $w=0.1445 \mathrm{eV}, \Delta_{\mathrm{eff}}=0.2 \mathrm{meV}$, and the effective potential $V=0.1 \mathrm{meV}$.

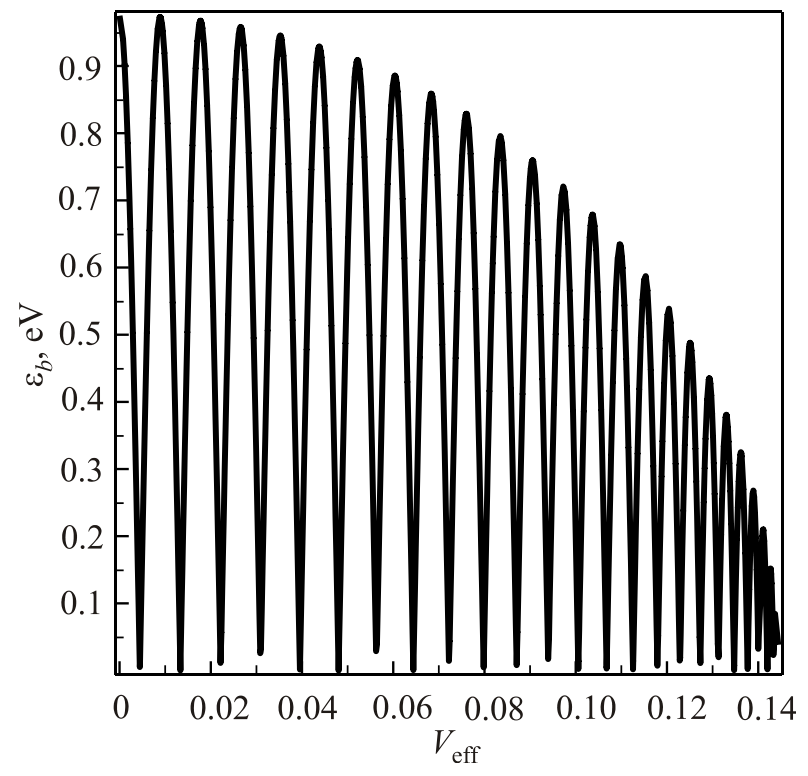

Fig. 2. The dependence of the energy of the bound state $\varepsilon_{b}$ in the Kitaev chain for the length of the chain $L=50$ (in units of interatomic distances in the chain) on the effective potential $V_{\text {eff. }}$ Other parameters are the same as in Fig. 1.

states for chains, and have shown that the Hamiltonian of the Kitaev chain can describe the experimental situation for the renormalized values of parameters. It has been shown how the energy of Majorana bound state depends on the size of the chain for large enough chain lengths. For small enough values of the effective potential such energy oscillates with the size of the system and with the value of the potential. For larger value of the potential there are no oscillations. Oscillations are caused by the real part of the wavenumber of the bound state. We have pointed out that for small values of the effective potential there exists a set of such values at which the energy of the Majorana edge mode is zero, i.e., the degeneracy of the ground state is restored. Real part of the wavenumber for the bound state becomes zero for large values of the potential. According to our estimates we expect that mentioned finite-length effects for the Majorana bound states can be observed in experiments (like scanning tunneling spectroscopy) in such magnetic chains on a superconducting substrate. We emphasize that finite-size devices with Majorana edge states are very important for practical applications for possible arrays of topological qubits. The effect of the applied effective voltage is also important from the viewpoint of perspective of manipulations with such sets of topological qubits.

Support from the Institute for Chemistry of the V.N. Karasin Kharkov National University under grant 4-15-13 (state registration number 0112U007569) is acknowledged. 
1. D.A. Ivanov, Phys. Rev. Lett. 86, 268 (2001); S. Das Sarma, D. Freedman, and C. Nayak, Phys. Rev. Lett. 94, 166802 (2005); A.Yu. Kitaev, Ann. Phys. (NY) 303, 2 (2003).

2. A.Yu. Kitaev, Phys. Usp. 44, 131 (2001).

3. L. Fu and C.L. Kane, Phys. Rev. Lett. 100, 096407 (2008); Phys. Rev. B 79, 161408(R) (2009).

4. R.M. Lutchyn, J.D. Sau, and S. Das Sarma, Phys. Rev. Lett. 105, 077001 (2010); Y. Oreg, G. Refael, and F. von Oppen, Phys. Rev. Lett. 105, 177002 (2010).

5. S. Nadj-Perge, I.K. Drozdov, B.A. Bernevig, and A. Yazdani, Phys. Rev. B 88, 020407 (2013); B. Braunecker and P. Simon, Phys. Rev. Lett. 111, 147202 (2013); F. Pientka, L.I. Glazman, and F. von Oppen, Phys. Rev. B 88, 155420 (2013); J. Klinovaja, P. Stano, A. Yazdani, and D. Loss, Phys. Rev. Lett. 111, 186805 (2013); S. Nakosai, Y. Tanaka, and N. Nagaosa, Phys. Rev. B 88, 180503 (2013); M.M. Vazifeh and M. Franz, Phys. Rev. Lett. 111, 206802 (2013).

6. V. Mourik, K. Zuo, S.M. Frolov, S.R. Plissard, E.P.A.M. Bakkers, and L.P. Kouwenhoven, Science 336, 6084 (2012); A. Das, Y. Ronen, Y. Most, Y. Oreg, M. Heiblum, and H. Shtrikman, Nature Phys. 8, 887 (2012); M.T. Deng, C.L. Yu, G.Y. Huang, M. Larsson, P. Caroff, and H.Q. Xu, Nano Lett. 12, 6414 (2012); L.P. Rokhinson, X. Liu, and J.P. Furdyna, Nature Phys. 8, 795 (2012).

7. J. Liu, A.C. Potter, K.T. Law, and P.A. Lee, Phys. Rev. Lett. 109, 267002 (2012); E.J.H. Lee, X. Jiang, R. Aguado,
Katsaros, C.M. Lieber, and S. De Franceschi, Phys. Rev. Lett. 109, 186802 (2012); D.I. Pikulin, J.P. Dahlhaus, M. Wimmer, H. Schomerus, and C.W.J. Beenakker, New J. Phys. 14, 12501 (2012); G. Kells, D. Meidan, and P.W. Brouwer, Phys. Rev. B 86, 100503 (2012); H.O.H. Churchill, V. Fatemi, K. Grove-Rasmussen, M.T. Deng, P. Caroff, H.Q. Xu, and C.M. Marcus, Phys. Rev. B 87, 241401 (2013).

8. S. Nadj-Perge, Y.K. Drozdov, J. Li, H. Chen, S. Jeon, J. Seo, A.H. Macdonald, B.A. Bernevig, and A. Yazdani, Science 346, 602 (2014).

9. J. Alicea, Rep. Progr. Phys. 75, 076501 (2012); C.W.J. Beenakker, Annu. Rev. Condens. Matter Phys. 4, 113 (2013).

10. P.A. Lee, Science 346, 545 (2014).

11. C.H. Lin, J.D. Sau, and S. Das Sarma, Phys. Rev. B 86, 22451 (2012); S. Das Sarma, J.D. Sau, and T.D. Stanescu, Phys. Rev. B 86, 220506 (2012); D. Rainis, L. Trifunovic, J. Klinovaja, and D. Loss, Phys. Rev. B 87, 024515 (2013).

12. J. Alicea, Y. Oreg, G. Refael, F. von Oppen, and M.P.A. Fisher, Nature Phys. 7, 412 (2011).

13. A.A. Zvyagin, Phys. Rev. Lett. 110, 217207 (2013).

14. E. Lieb, T. Schultz, and D. Mattis, Ann. Phys. (NY) 16, 407 (1961); P. Pfeuty, Ann. Phys. (NY) 57, 79 (1970); A.A. Loginov and Yu.V. Pereverzev, Fiz. Nizk. Temp. 23, 712 (1997) [Low Temp. Phys. 23, 534 (1997)]. 